\title{
MAAO, mémoire d'une organisation
}

In: Culture \& Musées. №2, 2003. pp. 101-127.

Citer ce document / Cite this document :

Eidelman Jacqueline, Monjaret Anne, Roustan Mélanie. MAAO, mémoire d'une organisation. In: Culture \& Musées. N², 2003. pp. 101-127.

doi : $10.3406 /$ pumus.2003.1180

http://www.persee.fr/web/revues/home/prescript/article/pumus_1766-2923_2003_num_2_1_1180 


\section{Résumé}

L'article rend compte d'une enquête réalisée auprès des personnels du MAAO à la veille de sa fermeture. L'utilisation de la méthode des récits de vie permet la constitution d'archives orales sur les différents corps de métier du musée. Mais la mémoire n'est pas l'histoire : ce récit pluriel est d'abord le « roman idéologique » d'un groupe spécifique à la veille de se dissoudre. Il apporte néanmoins des éclairages utiles sur l'organisation-musée : agencement des principales catégories d'activités (mise en collection et en exposition, veille et soins, administration et communication, accueil et médiation) ; manière dont les métiers se sont façonnés, diversifiés, qualifiés ou déqualifiés ; rapports hiérarchiques, professionnels et affectifs dans le contexte de travail et de hors-travail. Au-delà du cas particulier (héritage colonial encombrant, politiques directoriales désaccordées, tension art/ethnologie), l'étude témoigne du mouvement de professionnalisation et de la recomposition du service public qui, depuis cinquante ans, ont été les éléments structurants du changement de la sphère muséale.

\section{Abstract}

This article reports the results ofa survey of the staff of the MAAO on the eve of its closing. Use of the method of testimonials has allowed the construction of an oral archive involving the different positions within the museum. But memory is not history; this plural testimonial is first and foremost the " ideological novel " of a specific group on the eve of a dissolution. The study nonetheless provides useful insight into the organization of the museum : its principal activities (contributions to the collection and exhibitions, caretaking, administration and communication, welcome and mediation). It also shows how différent professions are constructed, diversified, qualified, or disqualified, in addition to recounting hierarchical, professional, and affective relationships in the workplace and outside the workplace. Beyond this particular case (burden of colonial heritage, disagreement between directors, the tension between art and anthropology) the study demonstrates the movement towards professionalization and the recomposition of public service, which over the lastfifty years have been the main impetus for change for museums.

\section{Resumen}

Presentamos aqui una investigación realizada con el personal del Musée des Arts Africains et Océaniens, justo antes de su cerradura. Utilizamos los " relatos de vida », que permiten obtener archivos orales tratando de diferentes oficios en el museo. Pero la memoria no es la misma cosa que la historia : estos relatos constituyen antes de todo el « relato ideologico » de un grupo especifico al momento de desaparecer. Ofrece informa- ciones importantes sobre la organizaciôn del museo (colecciones, exposiciones, administraciôn, comunicaciôn, mediaciôn, acojida) ; la diversificaciôn y las transformaciones de los oficios ; las relaciones jerarquicas, profesionales y afectivas. Allende el caso particular de este mueso colonial, la investigaciôn muestra el movimiento de profesionalizaciôn y los cambio en el servicio publico, cosas que influiron en los cambios del marco museal.

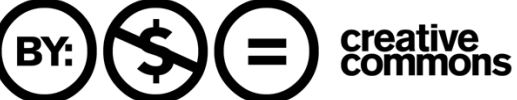


JACQUELINE EIDELMAN
$A N$ N E
$M O N J A R E T$
MELANIE ROUSTA N

\section{MAAO, MÉMOIRE D'UNE ORGANISATION}

Ue musée national des
Arts d'Afrique et d'Océanie
(MAAO) ferme ses portes le
31 janvier 2003 ; le transfert
des collections en direction
du musée du quai Branly a
commencé ; les personnels
se préparent à changer d'af-
fectation - la plupart n'iront
pas dans le nouveau musée.

C'est dans ce contexte qu'une équipe du Centre de recherche ir les liens sociaux (Paris-V/CNRS) a été sollicitée pour entrerendre un travail sur la "mémoire vivante " des agents, tous srps professionnels confondus, qui y ont travaillé ou qui y traiillent encore ${ }^{1}$.

Au fil des entretiens, des observations et d'une enquête par surrier ${ }^{2}$, la mémoire du MAAO s'est élaborée en croisant mémoire ttime et mémoire du lieu. Tantôt aux marges, tantôt au coeur, a discours sensible apparaît : c'est la mémoire qui se défie des śrités officielles, qui se soucie des sentiments. Capricieuse, multirme, elle ressasse, retourne sans cesse aux origines tout en cherrant à s'émanciper. Elle tente de se déprendre, mais adhère $₫$ lieu.

Mais la mémoire collective ne se fabrique pas seulement 1 fonction du passé, par interaction des souvenirs partagés " en mille ". Elle le fait aussi en fonction d'une conjoncture et de ses ijeux. L'annonce de la fermeture du musée a un effet de cristaliation des souvenirs et de libération de la parole : la chronique a l'institution est revisitée, les soubassements des crises ou les vénements déclencheurs reconsidérés, les régimes d'affronteients et de conciliations sondés une nouvelle fois.

Toutefois la mémoire n'est pas l'histoire. Il s'agit ici d'arlives orales qui réclameraient un traitement croisé avec des :chives administratives, des vérités factuelles... Tel quel, ce récit luriel est d'abord le "roman idéologique” "d'un groupe spécique. Parce que ce collectif est à la veille de se dissoudre, il père un retour sur une communauté de destin. Dans la situation 'entretien, celle-ci est mise en scène par chaque narrateur. Mais ans l'élaboration des souvenirs individuels, il ne peut y avoir lémoire sans identité, et inversement : le "mémorant "apprivoise : passé, se l'incorpore et le marque de son empreinte, et, par le 
fait même, opère une prise de conscience de soi dans la durée (Candau, 1996). De cette façon, la collection de biographies " en miroir " recueillies au MAAO rend manifeste l'un des mécanismes de la construction identitaire et, en particulier, dévoile les interactions entre identité institutionnelle, identité de métier et identité au travail (Dubar, 1991).

Notre posture méthodologique est donc décalée par rapport à celle du sociologue de l'entreprise qui procède à une lecture synchronique et formelle d'un système en actes ou à celle de l'ethnologue du travail qui observe et récolte les savoirs techniques et leur mode de transmission. C'est en mobilisant les identités plurielles et dynamiques issues de "l'étiquetage mémoriel " (Candau, 1996) que notre approche par récits de vie révèle l'organisation MAAO. Ainsi, à travers la reconstitution des trajectoires personnelles sont clarifiés les règles de l'établissement et les pratiques de fonctionnement, les rôles et les statuts, les principes de formation des groupes et l'arrangement des réseaux. Mais encore, des configurations problématiques se dégagent, des univers de justification s'éclairent (Boltanski et Thévenot, 1991). Enfin, par rapport aux recherches et publications existantes sur la professionnalisation des musées (Heinich et Pollak, 1989 ; Octobre, 1996 et 1999 ; Publics et Musées n 6, 1994 ; Poulard, 2002), nos résultats concrétisent les mouvements de fond qui ont touché la sphère muséale depuis l'entre-deux-guerres, formalisent les interactions entre identités et structure, délivrent des indicateurs tangibles de l'évolution des métiers et de ses motifs.

Dans cet article, nous montrerons comment le passage d'une tutelle à une autre et des politiques directoriales antinomiques ont engendré un ordre complexe. En détaillant les différentes catégories d'activités (mise en collection et en exposition, veille et soins, administration et communication, accueil et médiation) et leurs agencements successifs, nous illustrerons comment les métiers se sont façonnés, diversifiés, qualifiés ou déqualifiés. En dernier lieu, nous décomposerons les rapports hiérarchiques, professionnels et affectifs dans le contexte de travail et hors travail. C'est parce que notre analyse embrasse simultanément ces différents plans qu'elle rend compte du changement (Ballé, 2000).

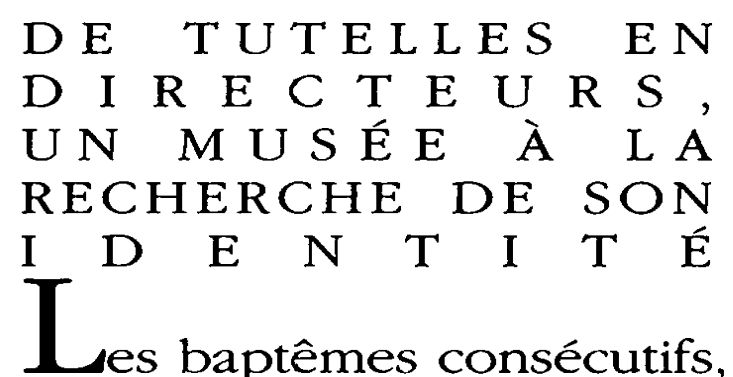

institutionnels divers et les différentes tentatives de restructuration du MAAO calquent l'histoire sociale et politique de la France dans 
ses relations avec les anciens protectorats et colonies, et, à travers eux, avec l'ensemble des cultures non occidentalest. Les philosophies d'action des directeurs successifs en sont fortement inspirées. Mais elles sont aussi le fait d'hommes de pouvoir qui ambitionnent de vrais changements, qui insufflent des projets à leur image, laissant derrière eux des traces matérielles comme affectives (Balandier, 1985). Nous appuyant sur la mémoire des personnels interrogés, nous débuterons l'analyse de cette quête d'identité en 1960, c'est-à-dire au moment où le musée passe de la tutelle du ministère des Colonies à celle du ministère des Affaires culturelles (pour la période 1931 à 1960 : Taffin, Blind et Martin, 2000 ; Taffin, 1999). Cinq principaux cycles structurent le temps institutionnel qui suivra, sept directeurs viendront façonner l'organisation du musée.

LA CONSTRUCTION D'UN VÉRITABLE MUSÉE : QU'EST-CE QU'UNE GUVRE?

Lorsque André Malraux prend sous son autorité les destinées du musée des Arts africains et océaniens, se veut symboliquement acquittée la dette du colonialisme et explicitement signifiée la reconnaissance de la valeur artistique intrinsèque des collections. Michel Florisoone a été nommé directeur de l'établissement en 1959. Il n'est guère préparé à mettre en application le postulat, cher à son nouveau ministre, de " l'impulsion sensible " lorsqu'il s'agit d'art et de muséologie et ne parvient pas à donner forme à l'idée de " l'esthétique à outrance " (Malraux, 1996)5. C'est également sur cette base que l'institution doit asseoir sa légitimité face au musée de l'Homme. En dépit d'efforts patients, Florisoone paraît échouer sur ces différents plans : il ne réussit pas à faire aboutir la refonte des espaces et de la muséographie, tire faiblement parti d'une campagne d'acquisitions qui profite prioritairement à des expositions hors les murs (Grand Palais, Orangerie, etc.), se trouve marginalisé par rapport aux débats intellectuels sur les relations entre arts primitifs et ethnologie (auxquels contribuent activement Claude Lévi-Strauss et Michel Leiris). Pourtant, un travail de longue haleine a été entrepris par les conservateurs : d'abord, le fonds originel est largement épuré de ses pièces " folkloriques "; ensuite, la constitution d'une véritable collection et l'organisation de son appareil scientifique sont en cours. Lorsque en 1973 Marguerite Olagnier-Riottot devient la directrice en titre (elle assurait, jusqu'alors la charge de conservateur du département Maghreb), les trois sections de l'Afrique noire, du Maghreb et de l'Océanie sont définitivement achevées, redéployées à travers le hall, les salles et les galeries, et installées dans des vitrines commandées aux ateliers du Louvre. Ce premier cycle s'achève donc sur un sentiment mitigé : le personnel conserve la nostalgie d'une exaltante ouvre collective (dont l'immense salle des Tapis, ouverte en 1977, constitue l'un des fleurons), mais il est conscient 
que les deux directeurs se sont insuffisamment souciés d'articuler identité des collections, fonction documentaire et action pédagogique faute d'un parti pris muséal affirmé.

UN MUSÉE AU SERVICE DES ÉCHANGES INTERCULTURELS : LE DÉVELOPPEMENT DE L'ACTION CULTURELLE

En 1979, avec une direction confiée à Henri Marchal, une nouvelle manière d'assumer l'héritage colonial se dessine : il s'agit de développer les échanges interculturels par une valorisation des collections et l'ouverture à l'art contemporain. À ces fins et entendant vivifier l'établissement, Marchal charge l'ADEIAO6, présidée par Lucette Albaret, de concevoir un plan d'action culturelle à l'intention d'un public large. Parallèlement, à l'Aquarium, la nomination de Michel Hignette comme directeur et un quadruplement des crédits permettent le lancement d'un programme de rénovation technique et muséographique. La fréquentation commence à " décoller ": dans les galeries d'abord, grâce au succès des expositions temporaires (la première est inaugurée en 1984, une dizaine d'autres vont suivre), des ateliers pour enfants, des activités et manifestations à destination des populations immigrées ; à l'Aquarium ensuite, avec le développement d'une stratégie de conquête et de fidélisation des publics familial et scolaire. Néanmoins, une rivalité s'amorce entre le personnel du musée, cantonné par son directeur dans des tâches de conservation et de gestion du quotidien, et les membres de l'ADEIAO, auxquels reviennent les missions " nobles " de réflexion et de réalisation des expositions. Les tensions s'exacerbent, l'ambiance devient délétère, la confusion est la règle. Hors des divisions statutaires, des engagements affectifs liés à la personnalité du directeur conduisent à des affrontements violents entre clans rivaux. La crise profonde est mise en exergue dans deux rapports de l'inspection générale des Musées de France.

UN PREMIER PROJET DE RESTRUCTURATION : LE MUSÉE COMME AMBITION COLLECTIVE

Démissionné une première fois, Marchal est remplacé, en juillet 1992, par Cécil Guitart qui se voit confier, par Jack Lang, une mission de réorganisation et de développement. S'ouvre une courte période d'espérance : l'ensemble du personnel est consulté pour la réalisation d'un projet de service qui s'appuie sur la nature du musée, la richesse de ses collections et la motivation retrouvée. Le discours muséal s'élabore autour de l'idée de " dialogue NordSud des cultures ", tentant une nouvelle fois de s'affranchir du passé colonial. Une première concrétisation en sera la mise en 
chantier de deux grandes expositions (avec le concours de la Réunion des musées nationaux [RMN] et de trois expositionsdossiers. Dans la même voie, la politique d'animation et de valorisation des collections est renforcée. Enfin, une réflexion sur la restructuration architecturale du bâtiment est amorcée en vue de transformer " le pavillon permanent des colonies en un musée fonctionnel ". L'embellie est brève et le programme qui aurait dû être mené à terme en trois ans est brusquement interrompu au bout d'une année : à la suite d'un arbitrage du tribunal administratif, Marchal est rétabli dans ses fonctions. Deux ans plus tard, le ministère le contraindra à démissionner une seconde fois. Alors que le collectif commençait à trouver son épanouissement dans une œuvre commune, ces allers-retours successifs le plongent dans le désarroi. Et ce sont les braises encore incandescentes d'un champ de bataille dévasté que le conservateur de la section Afrique, Étienne Féau, s'emploie à éteindre en tant que directeur intérimaire.

REGARDS CROISÉS ENTRE SOCIÉTÉS OCCIDENTALES ET NON OCCIDENTALES : LE PARTI PRIS DE L'ART CONTEMPORAIN

C'est à Jean-Hubert Martin que reviendra la tâche de remobiliser un personnel traumatisé. Nommé en 1994, le nouveau directeur infléchit la doctrine muséale une fois encore : la vocation du MAAO sera désormais d'affirmer que les sociétés non occidentales ne sont pas statiques et qu'il convient de porter sur elles un regard non stéréotypé. Cette idée-force, Martin l'a déjà éprouvée lors de l'exposition Les Magiciens de la terre présentée à la Grande Halle de la Villette et au centre Georges-Pompidou en 1989. Avec le concours d'une nouvelle génération de conservateurs, il va pouvoir lui donner toute son ampleur. Est d'abord élaboré un vaste programme d'expositions temporaires auquel la RMN, qui s'est définitivement substituée à l'ADEIAO, prend une part déterminante (seul exemple dans les musées nationaux, une coordinatrice est détachée au MAAO). Mais cette fois-ci, les forces vives du musée sont mobilisées. Dans la seconde moitié des années quatre-vingt-dix, plus d'une vingtaine d'expositions seront conçues qui mettent en perspective art contemporain et rehaussement des collections 7 . Ensuite est orchestrée une vigoureuse politique de communication avec développement du secteur événementiel et de l'action culturelle. Enfin certaines salles sont rénovées (la place de Danse de la salle Mélanésie, les parties centrales de l'Afrique et du Maghreb, avec en outre pour cette dernière deux salles latérales, la salle Australie) et les réserves restructurées sous le mot d'ordre de la conservation préventive. Désormais, la fréquentation du musée dépasse les trois cent mille visiteurs par an et le MAAO est devenu un musée " tendance ". Pourtant, la nouvelle 
forme d'organisation où sont privilégiés certains types de compétences produit des effets de clivages professionnels et statutaires.

\section{VERS LA FERMETURE : LES PASSEURS}

En arrière-plan, le projet d'un musée des Arts premiers menace ce fragile équilibre même s'il peut laisser espérer que le programme inachevé de refonte du musée va trouver une issue. Et c'est sur une ligne de crête particulièrement périlleuse que l'établissement, et au premier chef son directeur, doivent évoluer. Bientôt, il apparaît que ce dernier ne sera pas sollicité pour conduire les destinées du futur musée du quai Branly. Germain Viatte reprend les rênes du MAAO à l'automne 1999. Il engage le chantier du déménagement en ne mobilisant que très partiellement l'expertise acquise et développée par le service de la restauration. Il doit également définir la ligne muséographique du quai Branly. Le MAAO ne peut-il pas constituer un propice banc d'essai ? Les expositions La mort n'en saura rien et Kannibals et Vabinés n'ont-elles pas proposé des solutions inédites en matière de clés de lecture des collections ? N'ont-elles pas établi le caractère contre-productif de l'opposition art et ethnographie ? Les enquêtes de publics n'ont-elles pas souligné qu'émotion, choc esthétique, réflexion éthique et civique, découverte et reconnaissance composent les différents registres que les visiteurs mettent en correspondance (Eidelman et Gottesdiener, 2002 ; Eidelman, Gottesdiener et al., 2002) ? Mais le personnel n'a plus le coeur de reprendre la longue quête de sens qui a taraudé les générations précédentes quant à la " vocation douteuse " et au " destin funeste " du musée. Le collectif est définitivement désagrégé.

L'identité institutionnelle à laquelle renvoie la mémoire collective est constamment apparue comme brouillée. Identité sujette à polémiques, dès lors que les politiques directoriales successives ne sont pas parvenues à stabiliser l'image des collections, à la mettre en phase avec ce qui se passait dans l'époque. Identité entachée par un héritage encombrant que les milieux culturels ont fait perdurer en surnommant l'établissement le "Cayenne des musées de France ", ou encore le "Bat' d'Af " ou même " la verrue ". On ne peut s'étonner, dans un tel contexte, de la difficulté à mobiliser durablement un collectif de travail autour d'un projet fédérateur. Pourtant, derrière cette histoire perturbée, et audelà des crises qui ont marqué de manière récurrente la vie du musée, on décèle un second système d'interactions entre les individus et leur environnement. Ce régime d'action a pour cadre l'activité professionnelle proprement dite et les changements qui la traversent. 
le MAAO est un bon exemple des transformations complexes des métiers du musée et des identités de travail. Selon les époques, le personnel du MAAO a compté entre une quarantaine et une centaine d'individus. Fonctionnaires, contractuels, vacataires; personnel affecté à plein temps, mi-temps, mis à disposition, détaché : toutes les situations ont été présentes. Les récits de vie mettent en lumière les ajustements et les décalages entre définition de soi à travers un projet professionnel et catégories officielles produites au fil du temps : mémoire longue quand les emplois sont anciens et qu'ils ont subi de profondes mutations, mémoire du temps présent quand ils sont apparus récemment.

\section{Mise EN COLLECTION ET EN EXPOSITION}

Traditionnellement, les collections sont au cour du musée : elles font ce qu'il est, autant qu'il fait ce qu'elles sont ; elles sont à la fois ce qui est montré et ce qui ne l'est pas. Au MAAO, les collections emblématiques sont celles des ouvres qui lui donnent son nom. Pour partie, elles sont issues de la section des Arts indigènes datant des années trente. Le tournant institutionnel de 1960 permet leur doublement en volume et les dote d'une double cohérence, géographique et statutaire. Subsistent néanmoins des ambivalences quant à la position et au rôle des personnes travaillant avec et sur ces ouvres. Dans la première moitié du $\mathrm{XX}^{\mathrm{e}}$ siècle, amateurs d'art, marchands, ethnologues et conservateurs forment un entrelacs serré d'intérêts. La création du musée des Arts africains et océaniens, son intégration à la direction des Musées de France, puis son accès au rang de musée national signifient, pour certains membres de l'établissement, à la fois une émancipation du musée de l'Homme et des disciplines qu'il incarne, et une prise de distance avec les marchés de l'art. Mais les réalités vont " suivre " avec un temps de retard. En effet, les liens avec les marchés demeurent constants et la présence d'ethnologues et d'anthropologues dans l'établissement sera continue. Les autres fonds (celui de l'Aquarium, mais aussi les fonds historiques et de la bibliothèque) ne sont pas non plus exempts de complexité. Les collections aquatiques ont vu leur représentation évoluer : autrefois, le crédit scientifique se trouvait du côté des blouses blanches de " la section hydrobiologique " et l'expression d'usage était "sciences et techniques en bas, brocanteurs en haut ". Peu à peu, avec la montée en puissance de la notion d'art au sein de l'institution, elles seront définies par défaut comme " non-œuvres 
d'art " ou encore, selon une hiérarchie inversée, "collections vivantes "par opposition aux " collections tout court ".

Critères, modalités d'acquisition et traitement des ouvres sont fortement corrélés à ces évolutions et cadrent l'activité des conservateurs. Leurs fonctions se sont polarisées tantôt sur certaines tâches (étudier, classer, conserver, entretenir), tantôt sur d'autres (enrichir, exposer, médiatiser) ; selon les périodes, elles se sont développées souvent en coopération, parfois en concurrence, aussi bien à l'intérieur de l'équipe de conservation qu'entre elle et les autres services du musée. Ces balancements ne sont pas sans rapport avec les cultures professionnelles qui, depuis la fin des années cinquante, se sont illustrées à travers trois générations de conservateurs ${ }^{8}$.

Il y a la génération initiale, presque entièrement féminine, issue de la formation traditionnelle de l'École du Louvre, dotée d'une bonne connaissance muséale et livresque des anciennes colonies, et qui consacre tous ses efforts à un programme de constitution et d'organisation scientifique d'une véritable collection d'art distincte de celle, anthropologique, du musée de l'Homme. La décennie 1970-1980 voit accéder au musée une deuxième génération, plus masculine que la précédente, aux itinéraires universitaires variés articulant stages, travail de terrain et connaissance des cultures non occidentales ; elle permet que se déploient plusieurs secteurs d'activités jusqu'alors marginaux : la restauration, la muséographie, l'action culturelle. Dans la durée, ces deux groupes sont moins en rivalité qu'en complémentarité : au début, le passage de relais se fait dans un rapport de compagnonnage; ensuite, tout se passe comme si un éventail élargi de compétences était mis au service d'un établissement constamment à la recherche de ses finalités. Certes, il existe des effets de clivage: certains relatifs au souci de voir respecter une autonomie du travail sur son aire culturelle de prédilection, d'autres nés de la confrontation de stratégies de carrière divergentes (présence continue au musée au lieu de missions longues, publications et charges de cours). Mais sur la fin de cette période, une solidarité se reforme, aussi bien entre les membres de l'équipe de conservation qu'avec le restant du personnel, lorsqu'un directeur maladroit paraît avoir mis les impératifs d'une gestion rentable et de la notoriété audessus de tous les autres.

Avec les années quatre-vingt-dix arrive une troisième génération de conservateurs fraîchement émoulus de la récente École nationale du patrimoine. Jusqu'alors la situation avait été paradoxale, presque " injuste, puisqu'un grand musée national n'avait pas ses matières spécifiques dans les options du concours de conservateur ". Si un enseignement commence à être dispensé à l'École du Louvre dès les années soixante-dix, il faudra toutefois attendre la création de l'École nationale du patrimoine, en 1986, pour que les arts africains et océaniens fassent l'objet d'un enseignement 
de spécialité intégré à la formation et d'une épreuve pour l'obtention du titre de conservateur dans le domaine?. Des tensions identitaires se matérialisent autour de la question des statuts et de la précarité de certaines situations alors que, par le passé, la distinction entre fonctionnaires et contractuels ou entre les différentes classes de conservateurs n'en avait guère suscité. En outre, le " nouveau " conservateur se voit caricaturé comme "modèle d'excellence théorique "par un personnel technique qui se perçoit comme déconsidéré, se vit comme dévalorisé dans son statut social et professionnel. Les réunions de montage d'expositions se font particulièrement houleuses : l'un dénonce une culture du nontravail, l'autre s'en prend à une culture de l'expertise, un troisième déplore la fin de la culture des savoir-faire. La gestion de ces crises offre aux trois derniers directeurs du MAAO matière à réflexion quant au délicat problème de l'orchestration des compétences.

\section{VEILLE ET SOINS}

Surveillants, agents techniques des galeries et de l'Aquarium, service de la restauration constituent en quelque sorte les " sens du musée ". Ces groupes professionnels ont connu des changements importants sous le double mot d'ordre de la rationalisation des compétences et de la rentabilité des activités. La professionnalisation du secteur muséal s'est réalisée, pour les uns, par une technicité et une scientificité accrues des tâches, et, pour les autres, par une moindre contribution à la vie du musée et une déqualification progressive.

Les personnels de surveillance, de sécurité et de maintenance constituent tout à la fois l'entité la plus importante et la plus ancienne. À l'origine, elle est alimentée par les emplois réservés aux anciens militaires - on se souvient encore qu'un des premiers plombiers avait été marin et fait les quatre cents coups en Indochine, ou que l'équipe avait compté dans les années soixante jusqu'à trois générations de vétérans en même temps. Elle est souvent confortée par un réseau relationnel et familial. L'uniforme avec casquette était de mise chez les gardiens, le bleu chez les ouvriers. On les portait avec fierté, celle d'un statut social reconnu, celle de la mission de service public. On comptait sur l'éducation mutuelle pour progresser dans la carrière sous le contrôle d'un maître formateur (le responsable de l'Aquarium, le contremaître, le chef de la surveillance...). Un changement se dessine au début des années quatre-vingt, pour devenir décisif dans la décennie suivante. Métaphoriquement, la fin du "vêtement de travail " est ressentie comme un signe d'affaiblissement du " respect " entre les différents corps.

Plus concrètement, les activités se transforment, les hiérarchies se modifient, les voies d'accès évoluent : l'identité de métier n'est plus une donnée. C'est l'arrivée d'une nouvelle génération 
qui a passé un concours, qui profite avec appétit des stages de formation continue offerts par la direction des Musées de France pour changer de catégorie, qui, au cœur même des équipes, demande que soient privilégiées les compétences plutôt que l'ancienneté. C'est aussi un changement de dénomination des métiers : par exemple, on porte désormais le titre d'" agent technique d'accueil, de surveillance et de magasinage " ou de " technicien " (voire d'" ingénieur ") des " services culturels " (au total, ils sont actuellement une trentaine), quand on s'appelait autrefois gardien ou gardien-chef - comme pour faire écho à la complexification des systèmes de sécurité à mesure qu'ils deviennent électroniques puis s'informatisent.

Dans les sous-sols, il fut un temps où les personnels des ateliers et de l'Aquarium formaient une même communauté de travail. Souvent, ils avaient été recrutés comme agents de surveillance et affectés à l'un ou à l'autre des services à la suite d'un " arrangement " interne avec la direction. Cela n'est plus totalement vrai. Au service de muséographie, on obtenait son affectation comme menuisier, peintre, électricien, tapissier muni ou non d'un CAP spécialisé et ensuite " l'expérience faisait le reste "; au bout de quelques années, on se pensait artisan, on avait un métier, et le sentiment de pouvoir maîtriser une technique, de produire, de créer. À présent, la dizaine de personnes qui officient encore dans ces ateliers se sentent en déshérence : elles ont la sensation que leur métier leur échappe depuis que le musée confie certaines des tâches qu'elles assuraient par le passé à des entreprises extérieures. Alors que la polyvalence a longtemps été vécue positivement au nom de la solidarité entre les corps de métier, elle est progressivement devenue contrainte et synonyme de dissolution des compétences. On ressent cruellement la concurrence, on la juge déloyale. Le mouvement est en quelque sorte inverse pour les techniciens spécialisés de l'Aquarium. Nul ne subsiste de l'équipe des anciens. Ils sont à présent cinq, titulaires d'un bac +2 ou d'un bac +3 dans une discipline scientifique ou technologique, alors que, pendant bien des années, on pouvait prétendre au titre de soigneur en ayant été embauché comme gardien et en passant ultérieurement un examen sous forme de travaux pratiques devant une commission du Muséum présidée avec bienveillance par Théodore Monod. Leur expertise est reconnue dans tous les aquariums nationaux et souvent elle leur permet de progresser dans la carrière grâce à la mobilité.

Enfin, le service de restauration des objets est passé du quasibricolage à la science de la conservation préventive, et, en quelques années, ses animateurs en ont fait la référence majeure en matière de collections ethnographiques. Celui qui, progressivement, en est devenu le responsable avait choisi d'entamer un processus de formation de haut niveau qui l'a amené, en France comme à l'étranger, à découvrir les innovations techniques pour les adapter 
à la gestion physique des collections et restructurer entièrement les réserves. Pourtant, en dépit de cette excellence avérée (plusieurs centaines d'étudiants, de stagiaires et de professionnels se sont succédé sur une petite dizaine d'années auprès des quatre techniciens), la question statutaire n'a jamais été résolue et obère largement l'avenir des membres du service au moment où est entamé le départ des collections vers le futur musée du quai Branly.

\section{ADMINISTRATION ET COMMUNICATION}

On aborde le domaine de la gestion : celle du personnel et du budget, de la circulation des oeuvres et des travaux, de l'information et de la communication. Le secteur de l'administration est loin d'être replié sur lui-même : simultanément, il est transversal à tous les autres et ouvre le musée vers l'extérieur. L'entité est entièrement féminine.

Trois secrétaires générales ont conduit son action depuis le début des années quatre-vingt-dix - c'est à partir de 1991 que des postes de cette nature sont créés dans tous les musées nationaux pour seconder les chefs d'établissement. Outre cet aspect, la secrétaire générale assure la fonction de directeur des ressources humaines et d'intendant du bâtiment. Pour la gestion courante, deux secrétaires sont actuellement chargées des aspects administratifs et comptables du musée et une troisième de ceux de l'Aquarium ; une quatrième est détachée à l'action culturelle. Deux standardistes se relaient pour distribuer les appels vers tous les services ; ce sont elles également qui délivrent quotidiennement les clés des bureaux.

Avec l'extérieur, les relations sont multiples, souvent croisées : continues avec tutelle et partenaires (DMF, Réunion des musées nationaux, direction du Patrimoine, établissement public du musée du quai Branly, etc.), ponctuelles avec les entreprises qui interviennent à des titres divers dans le bâtiment, épisodiques avec les enseignants et autres responsables de visites organisées dont il faut planifier et régler la vcnuc. La circulation des ouvres et l'organisation des travaux sont l'affaire d'une régisseuse, interface entre les ateliers techniques, la surveillance et la conservation; la fonction est récente, la tâche est andue. Elle est parfois mal tolérée, synonyme d'une démultiplication des niveaux hiérarchiques et de la bureaucratisation des activités.

La communication est apparue depuis moins de dix ans : en partant de presque rien, il a fallu constituer un réseau de relais dans le monde de la culture et des médias (il s'élève à présent à quatre mille deux cents personnes et sept cent cinquante journalistes), doter l'établissement d'une identité visuelle (le logo " crocodiles "), orchestrer des campagnes de promotion... ou plus simplement tracer le chemin entre le métro et le musée. Il y a eu 
un effet "boule de neige " après qu'un célèbre couturier eut choisi la salle des fêtes pour présenter sa collection : défilés, tournages, reportages, publicités se sont enchaînés, popularisant l'établissement et lui amenant un nouveau flux de visiteurs.

Entre l'administration et les autres entités du musée, les rapports ont, selon les époques, été parfaitement décontractés ou extrêmement tendus. Ils se sont pacifiés à l'occasion des innombrables réunions durant lesquelles la collectivité est parvenue à concevoir et adopter le règlement intérieur du personnel d'accueil et de surveillance. Plus récemment, les bureaux du secrétariat général sont devenus une caisse de résonance de la détresse et des espérances des personnels : c'est là que se débattent les devenirs professionnels à l'aube de l'aventure périlleuse du quai Branly.

\section{ACCUEIL ET MÉDIATION}

Jusqu'aux années soixante-dix, le public ne se bousculait pas aux portes du MAAO ${ }^{10}$ et le service aux ouvres prenait le pas sur le service au public. Les activités de billetterie et de surveillance des visiteurs formaient l'essentiel du travail d'accueil. Avec un public peu nombreux et relativement homogène, la tâche était aisée même si, parfois, un peu ennuyeuse : "On surveillait les visiteurs comme on surveillait les ouvres."

Une politique d'expositions régulières et un programme d'embellissement de l'Aquarium vont dissiper cette indolence. La zone d'accueil est un poste d'observation privilégié des changements : les familles sont de plus en plus nombreuses, les groupes scolaires envahissent le hall, les touristes étrangers font leur apparition ; bref, le pèlerin nostalgique de l'époque coloniale ou l'amateur éclairé ne constituent plus les figures dominantes. Diversité, métissages : le public est pittoresque. Une image, un commentaire : "Une jeune maman blonde et à la mode, un papa rasta et un bébé noir et blanc "; ils viennent au MAAO plutôt qu'au Louvre parce qu'ils savent que c'est aussi un endroit de divertissement, parce qu'il y a les poissons, parce que les collections sont abordables, parce qu'il n'y a pas cette ambiance de musée où l'on chuchote, que c'est un endroit de grande liberté... À l'Aquarium, le surveillant s'improvise médiateur : il salue les enfants, les met à l'aise, tout en leur demandant de respecter certaines règles; il leur explique qu'il ne faut pas frapper les parois des bacs parce que "si on cogne sur la vitre c'est comme si on cognait sur le crâne et ça fait mal ; dans l'eau, le son court plus vite, il s'amplifie, il fait du mal ". Dans les galeries d'Afrique et d'Océanie, la conférencière fait asseoir les petits, explique longuement, répète lorsqu'ils n'ont pas saisi ; plus tard, elle les conduira aux ateliers de pratiques artistiques.

Depuis le moment où un véritable service culturel a été créé - bénéficiant de l'attribution par la Réunion des musées nationaux 
d'une équipe stable de conférenciers ainsi que de la mise à disposition de quelques enseignants par le ministère de l'Éducation nationale -, il existe un programme de visites guidées à l'intention des groupes ou des visiteurs individuels, et l'on trouve, dans les salles, des fiches pédagogiques qui s'adressent à un public élargi et, dans les expositions, des parcours ludiques qui répondent aux attentes des familles.

Parce que certains conservateurs sont convaincus qu'"il n'y a rien de faux dans la communication qu'on peut avoir avec les objets ", on a longtemps hésité sur la nature des clés d'interprétation qui doivent être livrées au visiteur. C'est essentiellement dans les expositions temporaires de la dernière décennie qu'ont été expérimentées différentes modalités de commentaires et de contextualisation. Pour autant, elles n'ont guère été transposées dans les galeries où la muséographie est demeurée réduite, pour l'essentiel, au simple appareil des vitrines épurées et des cartels classiques. De manière générale, on s'est refusé à courir le risque de transformer les cimaises en livre mural ou de figer les ouvres dans des dioramas, quitte à se détourner de la question des niveaux de lecture et de la scénographie des collections permanentes (exception faite des salles océaniennes et australiennes) ${ }^{11}$. En quelque sorte, on a renoncé à traiter le problème en portant tous les efforts sur l'action culturelle périphérique (les spectacles, les concerts, les conférences, les rencontres) plutôt que sur l'interprétation des œuvres in situ.

Cette même orientation trouve une autre illustration dans la refonte de la bibliothèque et de la librairie. La première offre désormais à la consultation plusieurs séries de dossiers (sur le bâtiment, les collections publiques et privées d'arts africains et océaniens, sur les manifestations organisées par le musée, sur le futur musée du quai Branly), de nombreux ouvrages consacrés aux arts contemporains extra-européens, et, sous certaines conditions, un fonds photographique et d'affiches anciennes. La seconde est, à la suite d'une complète réorganisation, non plus un modeste comptoir de vente, mais une véritable librairie d'art ; elle est visitée régulièrement par une clientèle d'habitués aussi bien que par le public tout-venant qui y trouve catalogues d'exposition, guide du musée et livres pour enfants ; elle a même contribué à la mise en place de salons de lecture au sein des expositions. Parce qu'il n'est plus concevable de ne prêter aucune attention aux demandes d'un " public insatiable ", au MAAO comme dans d'autres musées, la question de la médiation a eu un effet réflexif sur l'institution en tant que dispositif de socialisation de la culture (Eidelman et Van Praët, 2000).

Ainsi, à travers la restitution des mémoires, il est possible de cerner les principales transformations qui ont marqué l'ensemble de la sphère muséale : la valorisation de la formation sur le tas a cédé le pas à la certification des compétences, entraînant 
déqualification et précarité d'un côté, et cloisonnement des emplois et des tâches de l'autre ; la formalisation de fonctions inédites a donné naissance à de nouveaux métiers (secrétaire général, régisseur, concepteur et réalisateur de projets culturels, chargée de la communication) et à de nouveaux services (tel celui de l'action culturelle) ; une expertise scientifico-technique s'est développée dans différents secteurs (restauration, maintenance); il a été mis fin au système des emplois réservés aux anciens militaires (au MAAO, il a survécu, un temps, à la tutelle du ministère des Colonies); les services de la surveillance et de la sécurité ont découvert la mixité ; certaines activités traditionnelles sont désormais partiellement ou totalement soumises à l'externalisation (montage d'exposition, entretien du chauffage, nettoyage du bâtiment), etc.

Face à cette conscience des changements structurels, les mémoires invoquent un âge d'or où les collectifs étaient agrégés et les habiletés socialisées, où l'interdisciplinarité était la norme, où l'on portait moins d'attention aux positions hiérarchiques, où les différents métiers faisaient corps, faisaient cause commune. Au-delà de cette idéalisation existent néanmoins de multiples formes relationnelles qui incitent le collectif à se penser et se vivre comme "une famille dans sa maison ". Sous cet aspect, le MAAO garde un petit côté " à l'ancienne ". Il convient de s'interroger sur ces formes de sociabilité et leur influence sur les modes de construction de l'identité au travail (Sainsaulieu, 1977).

\section{UN "ESPRIT MAISON " \\ L' \\ effectif réduit de l'équipe (moins de cent} aujourd'hui), une certaine sédentarité de l'emploi, un investissement au travail, un attachement au lieu mais aussi des personnalités contrastées de directeurs sont des facteurs explicatifs d'un modèle de fonctionnement organisationnel et relationnel singulier.

Si le contexte a changé, si l'équipe n'est plus celle des premières heures, il reste que chez la plupart des membres du personnel s'exprime l'idée que l'identité institutionnelle a été stigmatisante. Est-ce l'intériorisation et le partage de ce sentiment qui nourrissent cet " esprit maison " qui fait du musée "une deuxième famille " autour de laquelle se cristallise une vie de travail, une vie au quotidien avec ses rythmes plus ou moins lents, plus ou moins linéaires, ses routines que l'on a du mal à bousculer? On est au musée un peu comme " chez soi ". On s'y imprègne en se formant, en prenant progressivement goût aux lieux et aux choses. On se mobilise ensemble sur des projets qui font dire qu'il existe et se reproduit ce qui s'apparente à une "culture d'établissement "(Cuche, 1996). On se démobilise tout autant, car la vie au musée n'est pas tous les jours facile. Et c'est bien cette alternance qui fait les temps du musée, des temps où 
le travail croise aussi le hors-travail, où les activités professionnelles et extraprofessionnelles s'enchevêtrent. Est-ce pour mieux rompre avec une monotonie qui définit souvent le temps au travail ? Est-ce pour s'accorder des moments de respiration ? Ou est-ce tout simplement pour améliorer le cadre de vie, pour faire corps pleinement avec le musée?

DE L'ESPACE DE TRAVAIL AU “ CHEZ-SOI "

En 1987, le bâtiment et son décor sont inscrits et pour partie classés au titre des monuments historiques. Ils n'en demeurent pas moins lieux de visite, de travail et parfois d'habitation. Malgré une apparence intemporelle ${ }^{12}$, les changements de parti pris muséographique, la modernisation des activités et l'évolution des métiers du musée ont profondément "travaillé " les intérieurs : redistribution des espaces (bureaux et ateliers, même si ces derniers, en sous-sol, ne seront jamais rénovés), installation des réserves (restructuration massive à partir de la fin des années quatre-vingt, mise aux normes de la conservation préventive sous ses formes les plus modernes, puis intégration du fonds historique), fermeture de certaines galeries et transformation d'autres en salles d'exposition temporaire. Un regard différent est alors possible, celui de la communion avec un lieu qui a été aimé " énormément ", dans lequel on a investi " beaucoup de son coeur, beaucoup de son âme ". L'intimité est physique avec le bâtiment, ses coins et recoins, au point qu'on se promet qu'un jour, on viendra avec " un podomètre ", tant il semble que " ce sont des centaines, des milliers de kilomètres qui ont été parcourus dans cette maison, à pied, dans les couloirs ".

Ce rapport au musée, presque viscéral pour certains, aurait conduit un agent à inscrire son nom sur une dalle de ciment frais. Cette signature pourrait apparaître comme une contremarque de la liste des héros de la conquête coloniale gravée sur l'une des façades du bâtiment. À tous les niveaux de la hiérarchie, on rcpèrc cc bcsoin de marquer son territoire par des signes personnels, de s'approprier son bureau ou son atelier (Monjaret, 1996). La production commence parfois par un réaménagement des locaux. Cloisonnements et décloisonnements dessinent les contours des espaces et des relations. Dans certains cas, le mobilier permet de recomposer des coins discrets. Ces aménagements ne suffisent pourtant pas à se sentir " chez soi ". Il faudra encore repeindre les murs et disposer les meubles, le matériel informatique. Dans les bureaux, tapis, bibelots, plantes vertes, photographies pourront s'ajouter au décor. Il arrive aussi que, dans certains locaux de travail, un réfrigérateur, un four à micro-ondes complètent l'équipement. Chacun s'entoure des objets qui font sens : cadeaux, souvenirs de voyage ou de pièces récupérées de l'ancien musée des 
Colonies. Le choix de ces objets et leur mise en scène disent les intentions des acteurs, informent sur leur symbolique. La dérision est un de ces modes d'expression - pour moquer l'image du musée, l'un des directeurs n'avait-il pas suspendu au-dessus de sa table de travail un crocodile naturalisé ? Il s'agit pour d'autres d'éviter l'écueil du stéréotype : tel agent de service technique a tenu à ne pas mettre d'affiches de pin-up sur les murs de son atelier pour rompre avec les habituelles représentations de l'ouvrier. Il en est de même avec le contenu sans alcool de son " frigo ". L'entretien des locaux de travail, des bureaux ou des ateliers, mais aussi des espaces réservés aux visiteurs entre dans ce processus d'appropriation du musée : il faut rendre propres ces lieux " poussiéreux ", " sales ", et métaphoriquement salis par l'histoire. Comment faire corps avec les lieux sans ce nettoyage, sans " laver son linge sale en famille "?

UNE “ GRANDE FAMILLE " DANS “ UNE PETITE MAISON "

L'intégration au musée peut se faire grâce à la mobilisation de réseaux d'affinité ou familiaux, de personnes travaillant déjà dans le milieu, en quelque sorte par cooptation. On entre soutenu par un père, " un frère de lait ", un cousin, un ami. Le jeune arrivant est alors pris en charge par les anciens qui le socialisent aux pratiques locales, aux normes du groupe d'appartenance. C'est ainsi que se tissaient et se tissent encore des amitiés générationnelles, des préférences entre groupes culturels : il y a les métropolitains et les autres, les Corses et les Antillais, les Martiniquais et les Guadeloupéens. Les accointances et intérêts professionnels apparaissent également : il y a les personnels de l'Aquarium, le corps de "gardiens " avec ceux de nuit et ceux de jour, les ouvriers au sous-sol et les autres. L'ambiance, pourrait-on se dire, est plus au cloisonnement qu'à la cohabitation. Pourtant, on se fait la bise, on se tutoie le plus souvent, et quand on se retrouve, on plaisante. Il y a eu des moments de véritables émulations collectives, d'autres moments où les groupes se sont constitués en clans opposés. Autrement dit, l'équipe se comporte comme une famille avec ses joies, ses tensions, ses drames ; avec ceux qui optent pour des attitudes de retrait, d'autres qui cherchent le consensus, sans que tout cela soit pour autant contradictoire avec le fonctionnement de la " maison " car ce qui les unit, c'est d'être et de travailler dans ce musée. Ils sont "fiers de leur musée ". Et comme dans toutes les familles, des personnalités se distinguent, jouent le rôle de ciment. Elles deviennent des figures de référence, des vedettes qui rejoignent le panthéon des directeurs. Elles détiennent la mémoire, le savoir ou l'esprit poète. Dans ces ambiances se nouent et s'entretiennent les liens, des liens de parenté même. Des couples se forment, d'autres se séparent ; le 
père surveille le fils. Vie privée et vie professionnelle se rejoignent. D'ailleurs, les relations entre collègues se poursuivent hors institution et sont au fondement de la sociabilité au sein de l'établissement.

\section{LE TEMPS DU TRAVAIL}

Au quotidien, la journée d'un musée tourne en boucle. Pour les visiteurs, les portes s'ouvrent à dix heures et se referment à dix-sept heures trente (pendant longtemps le musée et la bibliothèque ont été fermés à l'heure du déjeuner) ; elles sont entièrement closes le mardi. Mais pour les employés, la pulsation ne s'arrête jamais. Si les temps et les rythmes sont propres à chaque catégorie de personnel, ils sont largement subordonnés au temps et au rythme des collections. Pourtant, il ne faudrait pas croire que seule la collection vivante de l'Aquarium est animée ; que. seule elle mobilise une attention de tous les instants, jusqu'à faire se confondre activité muséale et " service hospitalier ". Les oeuvres, elles aussi, vont leur vie qui gouverne celles de leurs sentinelles. Certes pas entièrement de leur fait : tout au long de l'histoire du musée, elles n'ont cessé de se déplacer du musée vers l'extérieur et inversement (restitution des dépôts, prêts multiples, legs et dons), entre les différents services du musée, allant et venant des vitrines aux réserves (pour tris et inventaires, en fonction des options muséographiques) ou des étagères aux bureaux quand elles sont fichées, documentées... Calendrier et mouvement perpétuel des visiteurs et des objets ordonnent le scénario du travail des personnels. Entretien des salles, sécurisation des espaces, maintenance des vitrines, création d'expositions, publication de notices et de catalogues, accueil des visites de groupes, animation des ateliers, diffusion des programmes d'activités : voilà du visible simple qui apparaît presque d'emblée à l'œil du visiteur. Mais, sous la fausse évidence, agit de l'invisible compliqué, se trame de la complexité.

Alternance entre la surveillance nocturne, avec ses trois rondes et ses équipes qui se relaient une nuit sur deux sur des périodes de vingt jours, et la surveillance de jour où se succèdent séquences sédentaires et nomades... Début de matinée avec la distribution des clés, la relève du courrier, la réception des livraisons, la préparation des caisses, la vérification de l'éclairage des vitrines, le ménage des salles... Mobilisation en synergie des équipes : administration et gestion des personnels, organisation et contrôle des travaux, conception des dossiers pédagogiques, réalisation des expositions et des manifestations culturelles, stratégies de communication... Travail croisé en sous-sol, pour les activités de peinture, tapisserie, menuiserie, électricité, chauffage, plomberie... Travail de longue haleine, tels la rénovation complète de 
l'Aquarium, le plan de sauvegarde des collections, le classement du fonds documentaire, le récolement des objets, ouvrages, dossiers de presse, affiches, photographies... Travail dans l'anticipation à travers les projets d'acquisition, la préparation des missions de collecte, la mise au point du planning de surveillance, l'établissement du calendrier des visites, conférences et ateliers, la programmation des expositions... Modes de régulation sociale informels et formels : tenue du standard, information sur la fréquentation, commandes d'ouvrages, mais encore visite matinale de la directrice à l'ensemble du personnel dans les années soixante, passage de relais entre les surveillances de nuit et de jour, réunions de coordination quotidiennes, réunions de service hebdomadaires... La routine n'est pas que source d'ennui : elle est ce qui fait la trame d'une communauté de travail.

\section{VIVRE AU QUOTIDIEN : HARMONIE ET TENSIONS}

Au quotidien, le musée s'organise également autour du horstravail. Certaines activités extraprofessionnelles contribuent à donner la sensation d'être chez soi, favorisent les temps de la convivialité. Les pauses qui jalonnent la journée permettent les rencontres, des moments de respiration (Monjaret, 2001c). Quand il n'y a pas de cafetière, quand une envie de bouger se fait sentir, les agents se rendent au distributeur de boissons au niveau d'une des entrées de l'Aquarium. Boire le café matinal en compagnie de collègues signale la mise en route au travail. Les pauses reconduites tout au long de la journée permettent, elles, de rompre momentanément avec une tâche professionnelle en cours : elles sont des temps récréatifs nécessaires. Certains services, plus que d'autres, ont la réputation d'un accueil propice à l'échange, on y passe pour saluer ses occupants. Il existe aussi une salle de repos, celle-là même qui avait été installée pour l'ensemble du personnel et que les agents de surveillance se sont finalement réappropriée. Entre midi et deux, l'heure du repas sonne. Aujourd'hui fermée, l'ancienne cantine des PTT réveille la nostalgie. On s'y rendait en groupe, tous corps de métier confondus. La cuisine y était familiale, l'ambiance chaleureuse. La nouvelle cantine est située à plusieurs stations de métro du musée et ne présente pas de tels avantages : on ne la fréquente guère, préférant prendre son casse-croûte sur place ou déjeuner dans les petits restaurants de proximité. Les années passant, les pratiques se sont plutôt individualisées, même si des groupes d'affinité, des relations interpersonnelles se perpétuent grâce aux tajines du dimanche, aux petits repas à la menuiserie. Il est arrivé aux beaux jours de piqueniquer dans le bois de Vincennes, de courir à plusieurs autour du lac. Seuls les " pots " officiels donnés à l'occasion des voux, des départs à la retraite ou de l'inauguration d'une exposition réunissent 
l'ensemble de l'équipe, ou, plus exactement, ceux qui estiment normal de s'y rendre. Il faut ajouter les " pots " plus officieux qui se font dans la discrétion des locaux de travail et réunissent un groupe restreint pour fêter par exemple la galette des Rois (Monjaret, 2001b). Pour le personnel, la fête n'a, semble-t-il, jamais vraiment fait partie de son mode d'expression au sein de l'établissement. Est-ce parce que ses formes de convivialité s'expriment ailleurs ? Est-ce parce que les festivités appartiennent à un autre monde, celui des galas, des spectacles qui occupaient et occupent parfois encore la prestigieuse salle des Cinq Continents ? Néanmoins, en souhaitant l'organisation d'une "fête de fermeture ", certains manifestent leur besoin d'un rite de passage qui encadre socialement le changement.

À la convivialité répondent les rivalités, aux amitiés, des inimitiés. Encore un paradoxe qui définit ce musée en proie à des tensions cycliques. Il y a cet investissement au travail et cet attachement au lieu. Et, conjointement, il y a ce quelque chose d'indéfinissable, ce " quelque chose, peut-être qui collait un peu aux murs, quelque chose d'un peu négatif ". Certains diront : "Une vie détestable, un lieu magnifique. "Au fil du temps, les groupes d'affinité se sont transformés en clans, certains agents choisissant, pour se préserver, de se tenir à l'écart. Le climat est devenu lourd, les ragots pesants. Des animosités interpersonnelles que l'on rencontre partout, on est passé à des luttes intestines, à des conflits collectifs qui ont provoqué, dans des circonstances différentes, le départ de directeurs. Le personnel résiste aux changements organisationnels que la direction impose, réagit aux signes du pouvoir et aux formes d'autoritarisme. Dans ces conditions, certains membres de l'équipe ont décidé de faire front, de ne plus se rendre aux réunions, de se syndiquer, de se mettre en grève. Les conditions de travail se dégradent à en devenir insupportables, comme si dans ce musée, la vie ne pouvait être calme.

FAIRE CORPS AVEC LE MUSÉE : ASPIRATIONS ET INSPIRATION

Celui qui pénètre chaque jour dans ce bâtiment ne peut rester indifférent à son architecture monumentale, aux marques de l'histoire que la pierre a figées, aux objets majestueux, précieux ou plus modestes qui composent son décor. Il ne peut pas non plus demeurer indifférent à l'Aquarium et à sa collection vivante toute de lumière et de couleurs tropicales. Certains, plus curieux que d'autres, plus attentifs à leur environnement, choisiront d'en savoir plus sur les espèces de poissons, sur le sujet d'une exposition, de récolter des informations à la bibliothèque, d'écouter d'une oreille discrète le conférencier. Le lieu éveille la curiosité, mobilise les sens. L'autoformation peut se faire plus implicite, elle existe par le seul fait d'être sur place, de côtoyer les œuvres 
et de travailler sur elles. Les employés prennent progressivement goût aux ouvres. Certains auront plaisir à repasser dans les galeries comme pour mieux s'imprégner encore de ce savoir à portée de main. Conjointement à cette formation en solitaire et pour répondre à une demande, il fut un temps où certains conservateurs transmettaient leurs connaissances au " petit personnel ", formaient également les guides, les agents de surveillance. Échanger, apprendre ensemble participe à une éducation mutuelle qui se prolonge parfois hors les murs. Nombreux sont ceux qui ont évoqué le récent voyage en groupe au musée de Chartres dont le but était la visite d'une exposition sur les représentations des Kanaks. À cet intérêt collectif répond un intérêt individuel qui se traduit par la poursuite de cet éveil au-delà du temps au travail. Les vacances sont parfois l'occasion de parcourir le monde, de rencontrer des populations dont les seuls indices au musée se réduisent à des objets, des documents écrits et de confirmer, de cette façon, l'attrait pour certains pays, certaines cultures. À leur retour, ces démarches contribuent à conforter l'idée que les membres du personnel font corps avec le lieu, qu'il existe un principe de don et de contre-don entre eux et le musée. S'ils reçoivent, ils donnent aussi. Ils exprimeront parfois cet attachement par un legs, comme ce fut le cas d'un agent des services techniques qui remit " un objet qui lui venait de son grand-père " : "Il avait fait don d'un sabre kabyle d'Algérie, qui était un souvenir de famille. Il s'était détaché de quelque chose de précieux..."

Le musée n'est pas seulement source d'aspirations, il est aussi source d'inspiration, espace poétique. Les productions ne sont pas seulement institutionnelles, elles relèvent aussi des créations personnelles (Gérôme, 1984). Ainsi, l'agent de surveillance, l'ouvrier peuvent se faire poète, peintre ou sculpteur. Est-ce par effet de sensibilisation aux ouvres, à l'environnement et à l'histoire du musée? Est-ce un intérêt individuel pour la littérature et les arts plastiques qui les mène à produire, à créer au sein du MAAO comme à l'extérieur ? Est-ce une façon de réenchanter leur univers quotidien? Les employés parlent de leur vie, de leur vie au musée et laissent percevoir leur émotion. Dans l'atelier des électriciens, une fresque murale représente un ciel bleu sur lequel sont dessinés des oiseaux orientés vers la seule fenêtre du local et un soleil qui cache la lune, sorte d'éclipse inversée. Ce motif exprime aussi l'idée de liberté, peut-être celle que les agents n'ont pas toujours eue. Dans un atelier voisin, quatre tableaux abstraits ont été réalisés par le menuisier à l'aide d'objets de récupération (entre autres le pull de l'électricien, le bleu de travail du peintre), quatre compositions qui parlent du métier des collègues, de leurs conditions de travail. Chaque élément posé est choisi avec soin pour symboliser au mieux son propos. L'installateur propose dans son local des maquettes de ce qui pourrait être un jour des sculptures monumentales. D'autres préféreront les mots et les rimes 
pour exprimer leurs sentiments. Le texte de l'un d'entre eux est un véritable hymne : "Quand j'ai su que le musée était foutu, qu'ils l'avaient crucifié, j'ai écrit une belle poésie sur le mAAO. "Ces productions symboliques appartiennent aux expressions sensibles sur les lieux du travail.

Les agents nous rappellent ainsi que création et production ne sont pas antinomiques, que le champ des compétences individuelles excède celui des qualifications professionnelles, qu'il existe des domaines secrets où chacun trouve les moyens de construire un bien-être au quotidien. Au MAAO, "l'esprit maison " cimente l'identité au travail à travers l'imbrication des sphères professionnelle et privée au sein même de l'établissement.

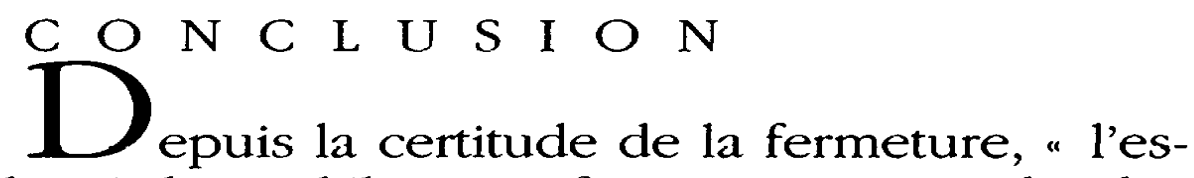
prit maison "a achevé de se déliter, renforçant une attitude plus individualiste, une prise en charge personnelle de son avenir professionnel et privé. Avec l'éclatement de la "famille ", c'est une relation passionnelle qui s'achève. Le roman collectif pourrait se résumer ainsi : "Coup de foudre, lune de miel, traversée du désert et rupture. Qui a dit qu'il n'y a pas d'amour heureux? "Ce destin émotionnel fort explique en partie les rapports humains difficiles voire traumatisants qui ont existé cycliquement, les "humiliations "subies tant par les directeurs que par le personnel et qui ont conduit à des drames, encore récemment.

Dans ce contexte, pour obtenir la confiance, pour délier la parole, notre démarche demandait une explicitation : il fallait faire la part entre une "commande sociale ", émanant de l'administration du musée, et une " demande sociale ", susceptible de s'exprimer chez l'ensemble des acteurs de l'établissement, notamment chez ceux qui peuvent ressentir leur " parole comme vaine " (Castel, 2002). C'est peut-être dans ce sens que nous avons assumé le rôle de " l'accompagnateur social "(Monjaret, 2001a), celui qui est à la fois le médiateur et le passeur, celui qui fait du lien aussi bien à l'intérieur du musée qu'entre l'institution et la société.

Au-delà du format de cette enquête de terrain, il se confirme que le musée est un miroir des changements sociaux, notamment des évolutions de métiers et en particulier dans le service public. Le double mouvement de disparition et d'apparition, de la déqualification et de la montée de l'expertise reconfigure progressivement l'univers des musées en tant que monde professionnel. Coexistent encore des formes anciennes et nouvelles de fonctionnement collectif et individuel : dans le travail et dans le hors-travail se perçoivent les signes et les cycles de ces changements, et, pardelà, les multiples modes de construction identitaire. 
Aujourd'hui, dans tous les musées, comme dans toutes les entreprises publiques ou privées, les identités au travail sont des manières de se situer dans la vie professionnelle et au-delà dans la vie sociale. Sur un demi-siècle, la mémoire des personnels du MAAO témoigne de ce passage entre une période où l'identification communautaire était la norme et celle où les identités s'individualisent. La dynamique qui va s'enclencher avec l'ouverture du musée du quai Branly ne peut ignorer ces transformations. À travers l'enquête sur la fermeture, elles auront été comme muséalisées.

J. E., A. M., M. R. Centre de recherche sur les liens sociaux (Paris-V/CNRS) 
NOTES

1. Cet article reprend, pour une large part, le contenu des textes de l'ouvrage MAAO-Mémoires (2002).

2. Le nombre d'entretiens compréhensifs s'élève actuellement à cinquante-cinq et le nombre de réponses à l'enquête par courrier à dix. Des visites et observations particulières ont complété la collecte des données.

3. Pour reprendre l'expression de P. Zylberman dans ses travaux sur l'histoire orale (article à paraître dans Etbnologie française).

4. De son inauguration en 1931 à nos jours, le musée de la PorteDorée aura changé de nom à plusieurs reprises : musée permanent des Colonies pendant la durée de l'Exposition coloniale, musée des Colonies et de la France extérieure en 1933, musée de la France d'outre-mer en 1935, musée des Arts africains et océaniens en 1961. Alors qu'il accède au rang de musée national en 1971 , ce n'est qu'en 1990 qu'un douzième département à la direction des Musées de France consacre la spécificité de ses collections : cette même année, il deviendra musée national des Arts d'Afrique et d'Océanie.

5. Selon Émile Biasini, ancien secrétaire d'État aux Grands Travaux du second septennat de François Mitterrand, il avait été chargé par André Malraux de la dévolution de l'établissement du ministère des Colonies à celui des Affaires culturelles.

6. ADEIAO : Association pour le développement des échanges interculturels au musée des Arts d'Afrique et d'Océanie.

7. En particulier, citons : Annette Messager ou la Galerie des cinq continents, pour l'art contemporain ; Vanuatu, pour les grandes expositions annuelles ; Asmat et Mimika d'Irian-Jaya, ou Ils sortent de leur réserve ou encore Coiffures sculptures d'Océanie, pour les expositions-dossiers.
8. Si dans son article dans la Revue françise de sociologie (op. cit.), Sylvie Octobre fait état de quatre générations de conservateurs, on n'en repère au MAAO que trois bien distinctes.

9. Le cas des conservateurs au service des arts maghrébins aura été différent en raison d'une institutionnalisation antérieure des arts islamiques et d'une distorsion plus grande entre le fonds, globalement très " ethnographique ", et la notion d'art.

10. D'après D. Taffin (1999), la fréquentation du musée des Arts africains et océaniens est de l'ordre de 140000 à 150000 visiteurs payants et gratuits entre 1966 et 1972 , en 1973 elle s'élève à 213965 visiteurs, en 1975 elle passe à 237000 visiteurs ; en 1983 elle sera encore de 234000 visiteurs. Ce n'est qu'à la fin des années quatre-vingt-dix qu'est franchi le seuil des 300000 visiteurs.

11. En dehors d'une enquête sur la composition du public de l'exposition Vanuatu et son évaluation sommaire (1994), il n'y a guère eu de souci de la réception des galeries et expositions jusqu'à l'enquête réalisée en 2000 à propos de l'exposition La mort n'en saura rien.

12. La campagne de photographies réalisée par B. Plossu l'illustre parfaitement (cf. MAAO-Mémoires, 2002). 
RÉFÉRENCES BIBLIOGRAPHIQUES

Balandier (G.). 1985. Le Détour. Paris : Fayard.

Ballé (C.). 2000. "Lès Musées, émergence d'un nouveau modèle ?", in Actes des journées d'étude: Publics et projets culturels. Un enjeu des musées en Europe. Paris : L'Harmattan.

Boltanski (L.), Thévenot (L.). 1991. De la justification. Les économies de la grandeur. Paris : Gallimard.

Candau (J.). 1996. Antbropologie de la mémoire. Paris : PUF (coll. "Que sais-je?").

Castel (A.). 2002. "La Réponse à la demande sociale ", in $\bar{A}$ quoi sert la sociologie?/sous la direction de B. Lahire. Paris : La Découverte.

Cuche (D.). 1996. La Notion de culture dans les sciences sociales. Paris : La Découverte.

Dubar (C.). 1991. La Socialisation. Construction des identités sociales et professionnelles. Paris : Armand Colin.

Eidelman (J.), Gottesdiener (H.) et al. 2002. Étude de réception de l'exposition "Kannibals et Vabinés *. CERLIS (CNRS / Paris-V) / établissement public du musée du quai Branly.

Eidelman (J.), Gottesdiener (H.). 2002. "Images de soi, images des autres : les modes opératoires d'une exposition sur des reliques d'Europe et d'Océanie ", in Patrimoine et Identité / sous la direction de B. Schiele. Montréal : MultiMondes / éditions du musée de la Civilisation.

Eidelman (J.), Monjaret (A.), Rous$\tan$ (M.). 2002. MAAO-Mémoires. (Photographies de B. Plossu.) Paris : Marval.

Eidelman (J.), Van Praët (M.) (dir.). 2000. La Muséologie des sciences et ses publics. Paris : PUF.

Gérôme (N.) (dir.). 1984. " Les Productions symboliques ouvrières *. Etbnologie française, 1984/2.

Heinich (N.) Pollak (M.). 1989. " Du conservateur de musée à l'auteur d'exposition : l'invention d'une position singulière ". Sociologie du travail, $\mathrm{n}^{\circ} 31$.

Malraux (A.). 1996 (1947). Le Musée imaginaire ( $3^{\mathrm{e}}$ éd. remaniée : 1965). Paris : Gallimard (coll. "Folio").

Monjaret (A.). 1996. "Être bien dans son bureau ". Ethnologie française, 1996/1.

Monjaret (A.). 2001a. " Fermeture et transfert de trois hôpitaux parisiens. L'ethnologue accompagnateur social ". Ethnologie française, 2001/1.

Monjaret (A.). 2001b. " La Fête, une pratique extraprofessionnelle sur les lieux de travail ". Cités, $\mathrm{n}^{\circ} 8$.

Monjaret (A.) (dir.). 2001c. " L'Alimentation au travail ". Cabiers Consommations et Sociétés, $\mathrm{n}^{\circ} 2$.

Octobre (S.). 1996. Conservateur de musée : entre profession et musée. Thèse 3 e cycle : EHEss.

Octobre (S.). 1999. " Profession, segments professionnels et identité : l'évolution des conservateurs de musée ". Revue française de sociologie, XI-2.

Poulard (F.). 2002. "Les Conservateurs de musée et le public ou les ressorts de l'intérêt pour les missions de médiation ". Musées et Collections publiques de France (sous presse).

Publics et Musées : "Professions en mutation ". 1994. $\mathrm{N}^{\circ} 6$.

Sainsaulieu (R.). 1977. L'Identité au travail. Les effets culturels de l'organisation. Paris : Presses de la Fondation nationale des sciences politiques.

Taffin (D.), Blind (C.), Martin (A.). 2000. "Pour une sociologie du musée colonial : pratiques et représentations du musée de la France d'outre-mer à travers la constitution de ses collections ", in $D u$ musée colonial au musée des cultures du monde / sous la direction de D. Taffin. Paris : Maisonneuve et Larose / musée national des Arts d'Afrique et d'Océanie. 
Taffin (D.). 1999. " Du musée de la France d'outre-mer au musée des Arts d'Afrique et d'Océanie (19601980) ", in Le Musée et les cultures du monde: actes de la table ronde des 8-9-10 déc. 1998. Paris : École nationale du patrimoine (coll. "Cahiers de l'École nationale du patrimoine "). 


\section{$L^{\prime}$}

article rend compte d'une enquête réalisée auprès des personnels du MAAO à la veille de sa fermeture. L'utilisation de la méthode des récits de vie permet la constitution d'archives orales sur les différents corps de métier du musée. Mais la mémoire n'est pas l'histoire : ce récit pluriel est d'abord le "roman idéologique " d'un groupe spécifique à la veille de se dissoudre. Il apporte néanmoins des éclairages utiles sur l'organisation-musée : agencement des principales catégories d'activités (mise en collection et en exposition, veille et soins, administration et communication, accueil et médiation); manière dont les métiers se sont façonnés, diversifiés, qualifiés ou déqualifiés ; rapports hiérarchiques, professionnels et affectifs dans le contexte de travail et de hors-travail. Au-delà du cas particulier (héritage colonial encombrant, politiques directoriales désaccordées, tension art/ethnologie), l'étude témoigne du mouvement de professionnalisation et de la recomposition du service public qui, depuis cinquante ans, ont été les éléments structurants du changement de la sphère muséale.

\section{$T_{h}$}

his article reports the results of a survey of the staff of the MAAO on the eve of its closing. Use of the method of testimonials has allowed the construction of an oral archive involving the different positions within the museum. But memory is not history; this plural testimonial is first and foremost the "ideological novel " of a specific group on the eve of a dissolution. The study nonetheless provides useful insight into the organization of the museum : its principal activities (contributions to the collection and exbibitions, caretaking, administration and communication, welcome and mediation). It also shows bow different professions are constructed, diversified, qualified, or disqualified, in addition to recounting hierarchical, professional, and affective relationships in the workplace and outside the workplace. Beyond this particular case (burden of colonial beritage, disagreement between directors, the tension between art and anthropology) the study demonstrates the movement towards professionalization and the recomposition of public service, which over the last fifty years bave been the main impetus for change for museums.

\section{$P$} con el personal del Musée des Arts Africains et Océaniens, justo antes de su cerradura. Utilizamos los " relatos de vida ", que permiten obtener archivos orales tratando de diferentes oficios en el museo. Pero la memoria no es la misma cosa que la historia : estos 
relatos constituyen antes de todo el " relato ideologico " de un grupo especifico al momento de desaparecer. Ofrece informaciones importantes sobre la organización del museo (colecciones, exposiciones, administración, comunicación, mediación, acojida) ; la diversificación y las transformaciones de los oficios; las relaciones jerarquicas, profesionales y afectivas. Allende el caso particular de este mueso colonial, la investigación muestra el movimiento de profesionalización y los cambio en el servicio publico, cosas que influiron en los cambios del marco museal. 